and non-smokers. The socio-cultural background needs to be considered when running public health campaigns due to differences in perception and responses to GHWL. Investigating the awareness of risks such as blindness, that have a low knowledge score but a high deterring impact, provides the chance to create a tailored approach when addressing this desensitisation.

\section{P289 CARBOXYHAEMOGLOBIN LEVELS IN EMERGENCY DEPARTMENT PATIENTS: AN IMPORTANT TOOL IN VALIDATING SMOKING HISTORY AND DETECTING "MISSED SMOKERS"}

RW Fowler. Queen's Hospital, Romford, UK

\subsection{6/thoraxjnl-2014-206260.407}

Introduction Blood gas analysers in emergency departments (ED) routinely report carboxyhaemoglobin $(\mathrm{COHb})$ levels, but unless environmental carbon monoxide poisoning is suspected, measurements are ignored, particularly when blood gases have been performed to check acid-base status. Raised initial $\mathrm{COHb}$ levels are likely to indicate highly nicotine dependent smokers needing specialist support to quit.

Methods Data were obtained from 824 samples analysed in the ED in June 2013. There were 82 samples with $\mathrm{COHb}$ levels $>2.0 \%$, including 66 from 55 identifiable patients. Only the first measurements were used. Records were requested and checked for diagnosis and smoking history.

Results 42 records were retrieved. The highest initial $\mathrm{COHb}$ level was $13.5 \%$; 15 had levels $>4.5 \%$. There were eight confirmed COPD and two possible cases. Eleven patients presented with drug and alcohol poisoning. Table 1 lists diagnoses.

28/42 (66.7\%) were current tobacco smokers; in 14 (33.3\%) pack-years could be estimated. There was no smoking history recorded in seven patients. Seven others were recorded as nonsmokers, but in five there was evidence to contradict this. One had an explanatory blood disorder, leaving one unexplained high level. Four patients smoked cannabis; all smoked tobacco cigarettes as well.

Only 4/42 (10\%) were referred to stop smoking services (SSS), though 10/42 (23.8\%) were already known and one was referred subsequently. Two previous quitters had evidently relapsed. Of the remainder known to SSS, two self-referrers quit, three failed and eight missed appointments.

\begin{tabular}{|c|c|c|c|c|}
\hline \multirow{2}{*}{$\begin{array}{l}\text { Abstract P289 Tabl } \\
\text { diagnosis }\end{array}$} & \multicolumn{4}{|c|}{ Carboxyhaemoglobin (\%) } \\
\hline & Total & $2-2.5 \%$ & $2.5-4.5 \%$ & $>4.5 \%$ \\
\hline COPD & 8 & 1 & 1 & 6 \\
\hline Cardiac & 5 & 1 & 3 & 1 \\
\hline Drug/alcohol poisoning & 11 & 2 & 4 & 5 \\
\hline Sepsis & 3 & 1 & 1 & 1 \\
\hline Acute abdomen & 3 & 1 & 2 & 0 \\
\hline Haemoptysis ? lung cancer & 2 & 0 & 1 & 1 \\
\hline Diabetic & 2 & 1 & 1 & 0 \\
\hline Others & 8 & 2 & 5 & 1 \\
\hline Total & 42 & 9 & 18 & 15 \\
\hline
\end{tabular}

In the largest group, those with drug or alcohol poisoning, smoking history was of poorer quality - pack-years could only be estimated in one case. Most had underlying mental health problems. None were referred to SSS.

Conclusions 1. Patients with $\mathrm{COHb}$ levels $>2.0 \%$ are usually tobacco smokers.

2. Multiple substance dependence is common. Most have mental health problems and are rarely referred to SSS - a missed opportunity to improve life expectancy in this vulnerable population.

3. Carboxyhaemoglobin levels must be included in a systematic approach to identify people needing intensive support to quit smoking.

\section{Screening and treating sleep apnoea}

\section{P290 VALIDATION OF PREOPERATIVE SCREENING ALGORITHM FOR OBSTRUCTIVE SLEEP APNOEA}

VM Macavei, J Mitic, M Berger, OE Mohr, TC O'Shaughnessy. Newham University Hospital, Barts Health NHS Trust, London, UK

\subsection{6/thoraxjnl-2014-206260.408}

Background Obstructive sleep apnoea (OSA) has been previously reported as a major risk factor for perioperative adverse events. ${ }^{1}$ Identifying patients with undiagnosed OSA can potentially have an impact on co morbidities and hospitalisation costs. $^{2}$

Aim To validate a previously reported screening tool for surgical patients suspected of having OSA.

Method A prospective study was performed in a university hospital between 1st Dec 2013 and 1st June 2014. An easy to use screening tool (STOP BANG) has been addressed to all patients prior to overnight oximetry sleep study during chest clinic assessment. The STOP BANG questionnaire incorporated 8 questions related to Snoring, Tiredness, Observed apnoeas, high blood Pressure, BMI $>30 \mathrm{~kg} / \mathrm{m}^{2}$, Age $>50$, Neck size $>15$ " and male Gender. Each affirmative answer was marked with 1 point. OSA was defined as dip rate $\geq 10$ events per hour associated with an oxygen desaturation $\geq 4 \%$ below baseline value.

Results A total of 102 patients have been included, 57 males $(55.8 \%)$ and average age $50.8 \pm 14$ years. 52 patients $(50.9 \%)$ have been diagnosed with OSA out of which 29 patients (28.4\%) had severe OSA (defined as dip rate $\geq 30$ events per hour).

Using logistic regression analysis, a STOP BANG score of $\geq 3$ had a sensitivity of $94.2 \%$ and specificity $72 \%$ with a positive predictive value of $77.8 \%$ and a negative predictive value of 92.3\% in detecting OSA patients.

Conclusion We have identified a high incidence of OSA of $50.9 \%$ in our sleep study population. We have validated STOP BANG questionnaire to be a useful predictor of OSA with a sensitivity of $94.2 \%$ and specificity of $72 \%$. This can be used during pre anaesthetic assessment indicating the requirement of chest clinic referral for sleep study at a score of $\geq 3$.

\section{REFERENCES}

1 Kaw, R, Chung, F, Pasupuleti, et al. Meta-analysis of the association between obstructive sleep apnoea and postoperative outcome. Br J Anaesth, 2012;109, 6:897-906

2 Mokhlesi, B, Hovda, MD, Vekhter, B, et al. Sleep-disordered breathing and postoperative outcomes after elective surgery: analysis of the nationwide inpatient sample. Chest, 2013;144, 3:903-14 